\title{
Diseño curricular: un esfuerzo de diez años de una red de universidades en Chile
}

\author{
Marco A. Villalobos-Abarca ${ }^{1}$, Raúl A. Herrera-Acuña ${ }^{1}$, José L. Contreras-Véliz ${ }^{2}$, y Marcela P. Varas-Contreras ${ }^{3}$ \\ (1) Facultad de Ingeniería, Depto. de Ingeniería en Comp. e Inf., Universidad de Tarapacá, Arica-Chile. \\ (Correo-e: mvillalo@uta.cl; rherrera@uta.cl) \\ (2) Depto. de Informática, Universidad Técnica Federico Santa María, Valparaíso-Chile. (correo-e: \\ jose.contreras@usm.cl) \\ (3) Facultad de Ingeniería, Depto. de Ingeniería Inf. y Cs. de la Comp., Universidad de Concepción, \\ Concepción-Chile. (Correo-e: mvaras@udec.cl)
}

Recibido Jun. 26, 2020; Aceptado Ago. 25, 2020; Versión final Oct. 20, 2020, Publicado Feb. 2021

\begin{abstract}
Resumen
El objetivo de esta investigación es dar una reseña de los proyectos ejecutados y los logros alcanzados durante 10 años de transformación curricular en la carrera de ingeniería informática en Chile. Se describe el trabajo realizado entre la Universidad de Tarapacá de Arica, la Universidad Técnica Federico Santa María en Valparaíso y la Universidad de Concepción. Se aplica la metodología de estudio de casos. Los resultados muestran que el desarrollo y ejecución de los proyectos por parte de esta red universitaria generó nuevos diseños curriculares, orientados por competencias y basados en proyectos, logrando así la institucionalización de procesos clave. Se concluye que realizar exitosamente proyectos de esta naturaleza y envergadura requiere del trabajo integrado de diversas instituciones de educación superior y el apoyo de recursos del estado.
\end{abstract}

Palabras clave: diseño curricular; competencia orientada; aprendizaje basado; proyecto; red; universidad

\section{Curricular design: a ten year Chilean University network effort}

\begin{abstract}
The objective of this research study is to summarize the process and achievements attained during a ten-year effort to transform the computer engineering degree curriculum in Chile. This study describes the processes performed by the Universidad de Tarapacá de Arica, the Universidad Técnica Federico Santa María, and the Universidad de Concepción. A case-study methodology is applied. The results show that the projects developed and executed by this university network generated new curricular designs that are guided by competencies and that are based on projects, achieving the institutionalization of key processes. It is concluded that to successfully perform projects of this nature and size requires integrated work by higher education institutions and governmental resources and support.
\end{abstract}




\section{INTRODUCCIÓN}

Las prácticas educativas, tanto a nivel nacional como a nivel mundial, están cambiando. Esto se debe principalmente a los nuevos paradigmas sociales y tecnológicos que presentan desafíos y oportunidades emergentes. La educación universitaria no está exenta de dichos desafíos, ya que se necesitan profesionales capaces de enfrentarse a un mundo altamente tecnologizado, donde los conocimientos estáticos y repetitivos ya no tienen una importancia preponderante, mientras que la capacidad de trabajo integrado, la innovación y el emprendimiento, son características cada vez más necesarias (García-Blanco y Sempértegui, 2018). Así, las universidades a nivel mundial han comenzado un proceso de rediseño de sus planes de estudios, para que la educación que imparten a sus alumnos, les permita integrarse al campo laboral rápidamente y puedan contar con las competencias necesarias para rendir en sus distintos roles (Tejada y Ruiz, 2016).

No sólo las prácticas, sino las políticas educativas están cambiando. Existe una creciente necesidad de minimizar la deserción de alumnos en las universidades, ya que, en general, ellas han permitido la movilidad social y el crecimiento de los países. Se hace necesario definir los aspectos que afectan la permanencia de los alumnos en sus respectivas carreras, para asegurar que puedan finalizarlas e integrarse a la sociedad como profesionales (Herrero et al., 2018). Estos aspectos deben ser considerados en los rediseños curriculares.

Casos nacionales e internacionales sobre métodos y experiencias particulares de rediseño curricular, se han documentado ampliamente. Por ejemplo, en la Tabla 1, se pueden apreciar trabajos que reportan experiencias realizadas en distintos países y disciplinas como ingeniería, medicina, educación, derecho, entre otras. El Estado de Chile no ha estado exento de esta realidad global y, por ello, también asumió el desafío de reformar su educación superior, para afrontar la realidad internacional y, en particular, los retos antes mencionados, para mejorar los resultados de sus programas de educación superior. En particular, apoyar esfuerzos para reformar los currículos de carreras más allá de un trabajo individual por universidad, sino mediante la formación de alianzas entre universidades chilenas. En este artículo, se presenta el caso de la carrera de Ingeniería Civil en Computación e Informática (ICCI) de la Universidad de Tarapacá (UTA), quien, en conjunto con la Universidad Técnica Santa María (USM) y la Universidad de Concepción (UdC), llevaron a cabo un amplio programa de rediseño curricular, con apoyo de recursos aportados por el Estado de Chile.

En el año 1998, el Gobierno de Chile, en acuerdo con el Banco Mundial (BM), a través del préstamo № 4404$\mathrm{CH}$, inició el diseño e implementación del programa "Mejoramiento de la Calidad de la Educación terciaria o Superior", conocido como MECESUP (http://dfi.mineduc.cl/). En el contexto del MECESUP, la planificación estratégica, la información para la toma de decisiones y los indicadores de desempeño (como retención y titulación estudiantil), pasaron a ser objeto de discusión pública y a formar parte de las condiciones establecidas por el Estado al momento de distribuir recursos de financiamiento en el sistema de educación superior.

En el año 1999, se realizó el primer concurso del Fondo Competitivo (FC), cambiando desde un sistema de asignación de recursos basados en criterios históricos, sin rendición de cuentas públicas, a un nuevo escenario de asignación basado en resultados. Los resultados del MECESUP incluyeron una mayor rendición de cuentas de los resultados en la asignación de recursos para la educación superior, el fortalecimiento institucional y el mejoramiento de la calidad y pertinencia de los programas, la contratación y formación de doctores y la ampliación del acceso para estudiantes de bajos ingresos, considerando la nivelación de competencias básicas de entrada. Así, con el MECESUP, se desarrollaron proyectos por distintas universidades. Surgieron asociaciones entre universidades en diversos puntos del país. La experiencia de dos proyectos conjuntos, desarrollados por la UTA, USM y UdeC, se relata a continuación.

Las universidades chilenas UTA, en el sector norte, USM, del centro y UdC, del sur, conformaron una red para abordar problemáticas comunes en sus carreras de Ingeniería en Computación e Informática. Los proyectos "Renovación Curricular en la Formación de Profesionales de Computación e Informática: Una Solución Articulada a Problemas Estructurales con Énfasis en la Movilidad Vertical y Horizontal" FSM0401 e "Implementación de Nuevos Currículos para Carreras de Ingeniería en Computación e Informática en Universidades Chilenas" FSM0711 fueron ejecutados por dicha red universitaria. El proyecto FSM0711 correspondió a la fase de implementación y operación de los diseños curriculares, logrados en el proyecto FSM0401. Estos apuntaron a resolver problemas transversales y específicos, detectados en las carreras señaladas, como: excesivo tiempo de permanencia en las carreras, baja pertinencia entre currículo y la sociedad, excesiva duración de los programas, falta de movilidad estudiantil, falta de consideración hacia los estudiantes, sus niveles de competencias al egreso y satisfacción por los servicios docentes, escasa articulación entre niveles de enseñanza al interior de los programas curriculares y al interior de las instituciones, e insuficiente aseguramiento de la calidad de servicios educacionales. Para resolver los problemas mencionados, los diseños curriculares deberían tener: perfiles de egreso que expresaran las 
competencias requeridas a profesionales en computación e informática en el ámbito laboral contextualizado en el sector productivo nacional, formación para el desarrollo de competencias para integrarse a su área laboral específica, estructura curricular flexible, sistema de gestión pedagógica que permitiera asegurar la calidad de la formación, uso intensivo de TICs en procesos educativos y de gestión curricular, entre otras.

Tabla 1: Trabajos que reportan experiencias en rediseño curricular.

\begin{tabular}{|c|c|c|c|c|}
\hline Autores & País & Disciplina & Necesidad Detectada & Objetivo \\
\hline $\begin{array}{l}\text { Jensen y } \\
\text { Lassen (2020) }\end{array}$ & $\begin{array}{l}\text { Dinamarc } \\
\text { a-Uganda }\end{array}$ & Pedagogía & $\begin{array}{l}\text { Incapacidad de los alumnos para } \\
\text { desarrollar proyectos. }\end{array}$ & $\begin{array}{l}\text { Rediseño del currículum en base a } \\
\text { "Aprendizaje Basado en Proyectos". }\end{array}$ \\
\hline $\begin{array}{l}\text { Ellahi et al. } \\
(2019)\end{array}$ & Pakistán & Ingeniería & $\begin{array}{l}\text { Falta de capacidad de } \\
\text { académicos y alumnos para } \\
\text { abordar tecnologías emergentes. }\end{array}$ & $\begin{array}{l}\text { Rediseñar el currículum y prácticas } \\
\text { para estar alineados a la industria } \\
4.0 \text {. }\end{array}$ \\
\hline Rata (2019) & $\begin{array}{l}\text { Nueva } \\
\text { Zelanda }\end{array}$ & Ingeniería & $\begin{array}{l}\text { Falta de capacidad en los } \\
\text { currículos actuales para manejar } \\
\text { sus contenidos en base a } \\
\text { conocimientos y habilidades. }\end{array}$ & $\begin{array}{l}\text { Aplicar la metodología de } \\
\text { Coherencia de Diseño de Curricular. }\end{array}$ \\
\hline $\begin{array}{l}\text { Martínez et al. } \\
\text { (2019) }\end{array}$ & Ecuador & Educación & $\begin{array}{l}\text { Desconocimiento de elementos } \\
\text { claves para realizar rediseño } \\
\text { curricular. }\end{array}$ & $\begin{array}{l}\text { Generar una base de conocimientos } \\
\text { para llevar a cabo el rediseño } \\
\text { curricular en carreras de educación } \\
\text { de forma exitosa. }\end{array}$ \\
\hline $\begin{array}{l}\text { Kim et al. } \\
(2019)\end{array}$ & $\begin{array}{l}\text { República } \\
\text { de Corea }\end{array}$ & Ingeniería & $\begin{array}{l}\text { Falta de concordancia entre lo } \\
\text { enseñado en las universidades y } \\
\text { las necesidades de la industria. }\end{array}$ & $\begin{array}{l}\text { Aplicar los preceptos de los } \\
\text { Estándares de Competencia } \\
\text { Nacional a carreras de ingeniería. }\end{array}$ \\
\hline $\begin{array}{l}\text { Novak et al. } \\
(2019)\end{array}$ & USA & Medicina & $\begin{array}{l}\text { Necesidad de actualizar los } \\
\text { conocimientos de los alumnos de } \\
\text { pregrado. }\end{array}$ & $\begin{array}{l}\text { Analizar las maneras en que las } \\
\text { escuelas de medicina pueden } \\
\text { actualizar sus currículos. }\end{array}$ \\
\hline $\begin{array}{l}\text { Ryan et al. } \\
(2019)\end{array}$ & Irlanda & Farmacia & $\begin{array}{l}\text { Generar un programa de } \\
\text { postgrado basado en } \\
\text { competencias. }\end{array}$ & $\begin{array}{l}\text { Desarrollar un programa de } \\
\text { postgrado, integrado al pregrado y a } \\
\text { las necesidades del mercado laboral }\end{array}$ \\
\hline $\begin{array}{l}\text { Icarte y Labate } \\
(2016)\end{array}$ & Chile & $\begin{array}{l}\text { Ingeniería } \\
\text { Informática }\end{array}$ & $\begin{array}{l}\text { Necesidad de mejorar de las } \\
\text { competencias de los alumnos }\end{array}$ & $\begin{array}{l}\text { Generar una metodología de diseño } \\
\text { curricular basado en competencias }\end{array}$ \\
\hline $\begin{array}{l}\text { Hurtubise y } \\
\text { Roman (2014) }\end{array}$ & $\begin{array}{l}\text { Estados } \\
\text { Unidos }\end{array}$ & Medicina & $\begin{array}{l}\text { Falta de "aprendizaje } \\
\text { significativo" en los alumnos }\end{array}$ & $\begin{array}{l}\text { Generar en los educadores las } \\
\text { competencias para entregar } \\
\text { "aprendizaje significativo" }\end{array}$ \\
\hline $\begin{array}{l}\text { Marsh et al. } \\
(2017)\end{array}$ & $\begin{array}{l}\text { Estados } \\
\text { Unidos }\end{array}$ & Farmacología & $\begin{array}{l}\text { Generar un modelo que mejore el } \\
\text { aprendizaje conceptual en los } \\
\text { alumnos. }\end{array}$ & $\begin{array}{l}\text { Incorporar aprendizaje activo en el } \\
\text { currículo. }\end{array}$ \\
\hline
\end{tabular}

EI FSM0711 contempló la ejecución de las siguientes actividades: a) Organización y planificación del proyecto, b) Revisión y afinamiento del diseño, c) Formación de competencias pedagógicas en profesores y ayudantes, d) Creación y adecuación de infraestructura, e) Generación de material didáctico, f) Generación de procesos para gestión, seguimiento y aseguramiento de la calidad del aprendizaje, g) Institucionalización y adecuación de aspectos reglamentarios y administrativos, h) Diseño del proceso de transición y puesta en marcha, y i) Creación de mecanismos para revisión y actualización de currículos. Estas acciones requirieron de especialistas en materias curriculares y docentes, la confección de material didáctico y el desarrollo del sistema de gestión curricular, que se gestionaron mediante la contratación de asistencias técnicas nacionales e internacionales. Además, se realizaron pasantías universidades destacadas por la calidad de sus ingenieros y sus currículos innovadores, orientados a la formación de competencias como emprendimiento, innovación y liderazgo.

\section{MÉTODO}

Se aborda la transformación curricular, en el contexto de la ejecución de los proyectos MECESUP FSM0401 y FSM0711, describiendo lo realizado y logrado durante 10 años (2010-2019). Dicha transformación, contó con la participación de los grupos académicos integrados por docentes, graduados y estudiantes, y otros actores, por ejemplo, empleadores y personajes destacados de la industria del software. La estrategia de investigación se enmarcó en la corriente cualitativa (secuencia de procesos). Los tres componentes más importantes de este tipo de investigación son los datos, cuyas fuentes más comunes son las entrevistas y la observación; los diferentes procesos analíticos e interpretativos de estos datos, para arribar a resultados o teorías, y los informes escritos o verbales (Tracy, 2019). El proceso por el cual se desarrolló el proceso en sí dentro de los proyectos, consistió en las siguientes etapas: (a) Observación de Experiencias - visitas a distintas universidades, Universidad de Sherbrooke (Canadá), Universidad de Texas (EI Paso, USA), Instituto Tecnológico de Monterrey (Monterrey, México) y el University College London (Londres, Inglaterra), y las Universidades de Los Andes, de Medellín, Politécnico Grancolombiano (Colombia); (b) Capacitación en temas de diseño curricular curricular y metodologías activas; (c) Asistencias Técnicas; (d) Generación de Normativas; (e) 
Renovación de equipamiento y adecuación de infraestructura.

El diseño de investigación elegido fue el estudio de caso (George, 2019). Se fundamenta en un análisis de las relaciones entre muchas variables concentradas en el caso, lo que dio lugar a la búsqueda de múltiples fuentes de evidencia. Se apoya fuertemente en entrevistas planteadas alrededor de pocos conceptos, las que se van desarrollando a lo largo del trabajo de campo realizado. Las técnicas elegidas, en concordancia con la estrategia y diseño planteados son: (a) Análisis de contenido de fuentes secundarias y específicas de la unidad académica, tales como: Informes parciales y finales emitidos al Ministerio de Educación (MINEDUC), anteproyectos o informes preliminares curriculares, plan de estudio de la carrera ICCI, programas de asignaturas, entre otras. Dado que el análisis es de contenido, se trató de una técnica de interpretación de textos, ya sean escritos, grabados, filmados y también el análisis de las transcripciones de las entrevistas realizadas; y (b) Entrevistas, personales, semiestructuradas a docentes que participaron de la ejecución de los proyectos MECESUP FSM0401 y FSM0711.

El estudio de caso se trata de una herramienta especialmente aplicable para este proceso, ya que se intenta reconstruir acciones pasadas, estudiar representaciones sociales y discursos de grupos. Las entrevistas se desarrollaron durante el primer semestre de 2019, participando 10 académicos que estuvieron directamente involucrados en la creación, ejecución y evaluación de los proyectos. Entre los académicos se encuentran los representantes de cada universidad de la red. A continuación, se describe el proceso de desarrollo de los proyectos para la UdeC, USM y se analiza en mayor detalle el caso de la UTA, por cual se inicia con ella.

\section{Universidad de Tarapacá - UTA}

Este caso se describe en dos grandes etapas: (1) el perfil de egreso, los principios de diseño y organización del currículo; y (2) la evolución hacia el diseño definitivo.

\section{Etapa 1 - Perfil de egreso, principios de diseño, organización del currículo}

El perfil de egreso fue uno de los primeros productos de las asistencias técnicas del proyecto MECESUP FSM0401. El proceso fue sistemático y consideró, en primer lugar, un marco general para el diseño del currículo. Se optó por diseñar un currículo orientado al desarrollo de competencias en vez de un currículo por competencias (modelo "puro") (Romero y González, 2016). El método de trabajo se basó en el análisis ocupacional, fue desarrollo por equipos de trabajo, los que se constituyeron por personas relacionadas con el ámbito de estudio, pero que ejercían diversas funciones o roles en él. Así mismo, se consultaron diversas fuentes de información, tales como marco país, marco universitario, mercado laboral, actores, literatura, normativas y leyes. Se desarrollaron instrumentos tales como encuestas, cuestionarios, y matrices de análisis documental. Además, se realizaron análisis de textos y declaraciones de actores, entrevistas y grupos focales.

Perfil de Egreso. Como resultado inicial, se derivaron categorías generales de competencias (Comunicación, Investigación, Producción Económica, Responsabilidad Social, Educación Permanente, Gestión, Habilidades Digitales, Multidisciplinaridad, Emprendimiento, Proyectos, Flexibilidad y Sensibilidad estética) y ejes transversales que debían contenerse en el currículo. Posteriormente, se redactaron las competencias, se validaron localmente (académicos), se validaron externamente a través de una asistencia técnica y, por último, se redactó la versión final.

Principios de diseño del currículo. De acuerdo a lo expresado por Bédard et al (2012), una de las críticas hacia la formación de los ingenieros, es su insuficiente habilidad en el diseño y su deficiente preparación en la utilización de los conocimientos científicos, matemáticos y analíticos para la creación de componentes, sistemas y procesos. Esto se debe fundamentalmente a que los currículos tienden a fragmentar los conocimientos en disciplinas y no consideran procesos de integración. Así, en el diseño del nuevo currículo, se consideró una formación orientada al desarrollo de competencias (como la innovación, el liderazgo y el emprendimiento) y al trabajo en equipo, para satisfacer las necesidades de la sociedad, considerando el sector productivo, políticas de desarrollo y ambiente globalizado. Para alcanzar el objetivo, se requiere de un cambio de prácticas pedagógicas con el propósito de atenuar las falencias de los programas actuales. Así Bédard et al. (2007) sugieren que buenas prácticas serían el uso del aprendizaje basado en problemas, el método de casos y el enfoque por proyectos, ya que se encuentra consolidado su uso tanto en Medicina como en Ingeniería. En base a las reflexiones anteriores, se establecieron los principios rectores que guiaron el diseño del nuevo currículo, que sea: dirigido por competencias, integrado-estructurado en semestres, alrededor de temas, orientado a proyectos, y con enseñanza Just-in-Time (JiTT) (Martin et al., 2018).

Organización del currículo. Los proyectos sirven para enfatizar dos funciones: integración de conocimiento y diseño. En el currículo, en la primera parte del plan de estudio, se pone mayor énfasis en la integración de conocimiento. La importancia de esto, es que el estudiante deberá centrarse inicialmente en la adquisición e integración de conocimiento de las ciencias subyacentes y del núcleo de los fundamentos de la ingeniería. 
Así, cada semestre en un nuevo plan de estudios, debería estar organizado alrededor de un proyecto, el que debe alimentarse con el contenido cubierto en todos los cursos y, respectivamente, los cursos deben apoyar al desarrollo del proyecto, de forma tal que directamente ayuden a los estudiantes a realizarlo. Esta "retroalimentación", normalmente, se convierte en uno de los principales incentivos para que los estudiantes puedan aprender y participar en las actividades propuestas en clase por medio del proyecto. Pese a la importancia de la adquisición de conocimientos técnicos y el razonamiento que normalmente son impartidos a través de las clases formales, existe la necesidad de proponer un ambiente de aprendizaje y un curso que permitan a los estudiantes participar en actividades complementarias que requieran habilidades y actitudes.

La adquisición de los conocimientos teóricos y prácticos, más aún en un plan de estudios orientado a competencias, impulsa la integración de la información adquirida. La integración representa un esfuerzo por contextualizar los conocimientos que se adquieren. La capacidad para integrar el conocimiento es una habilidad que los estudiantes deben desarrollar a lo largo de su carrera. En un programa de estudios impulsado por competencia, los temas deben sustentarse en la realidad profesional y reflejar el perfil. La secuencia de temas a lo largo del plan de estudios, debe reflejar una "curva de crecimiento profesional". Así, que se refiere al tema presentado en cada semestre, este ayuda a los estudiantes a ver y hacer los vínculos entre el contenido enseñado en clase y las competencias. Las competencias, que son los objetivos en los perfiles, pueden ser alcanzadas en la realización de un proyecto que está directamente relacionado con el tema.

\section{Etapa 2 - Evolución hacia el diseño definitivo}

El currículo evolucionó desde un diseño preliminar, pasando por un diseño de transición y hasta llegar a un diseño definitivo.

Diseño Preliminar. Se diseñaron matrices que relacionaron las categorías descubiertas entre el proceso de diseño del perfil y las competencias, pero se contextualizaron con las competencias del CDIO (Crawley et al., 2011). Posteriormente, para las actividades curriculares (cursos) se consideraron las propuestas de la ACM-IEEE, UNESCO, Libro Blanco Español sobre currículos de Ingeniería Informática, Ingeniería de Software, entre otros. Así, considerando los principios rectores, una organización entorno a proyectos y las propuestas de contenido indicadas anteriormente, se derivaron tres aproximaciones, definiendo un primer layout para la malla curricular de la carrera ICCI. Esta configuración, otorgaría como salida intermedia el título de Ingeniero de Ejecución en Computación e Informática, al $8^{\circ}$ semestre. También, otorgaría el grado de Licenciado en Ciencias de la Ingeniería y opcionalmente, cumpliendo con requisitos específicos, el grado de Magíster en Ingeniería de Software (profesionalizante). Por otra parte, se incorporaría en el primer semestre una nivelación en ciencias básicas y competencias básicas. El último semestre se dedicaría mayormente a la actividad de titulación e incorporaría dos prácticas profesionales, una de ellas dentro del $8^{\circ}$ semestre, con una supervisión directa.

Una vez definida la malla curricular, se procedió a desarrollar los contenidos de los cursos, teniendo en consideración las subcompetencias y una matriz de tributación, donde se indica la intensidad, en un rango de 0 a 3, del aporte de cada uno de los cursos al logro de las subcompetencias. Respecto de la evaluación de las competencias a ser alcanzadas o logradas por los estudiantes, se consideraron tres momentos, al quinto, al noveno y al doceavo semestre de la carrera. Esto se debe a que: Al término del primer momento de evaluación de competencias, el estudiante debería ser capaz de resolver problemas de mediana complejidad, aplicando un método de desarrollo de software. Al término del segundo momento, el estudiante debe ser capaz de poner en marcha un proceso de desarrollo de software, basado en un modelo de proceso, en el contexto de un proyecto. Por último, al momento de egreso, el estudiante debe ser capaz de incorporar las tecnologías de información como herramientas estratégicas, para mejorar los procesos de negocios en una organización. Para llegar a la malla curricular definida, sería necesario generar un proceso de transición, basado en una malla curricular intermedia que permitiera hacer los ajustes necesarios, tanto a nivel de conformación de cursos como a nivel de normativas institucionales.

Diseño de transición. Durante el año 2013, se puso en marcha un plan de estudio que considera la mayoría de las bases iniciales, en particular, una malla curricular organizada alrededor de proyectos. Sin embargo, aspectos importantes que eran la nueva forma del currículo (enseñanza Just-in-Time) disposición de los cursos en cada semestre, evaluación semestral, entre otros) y la implementación de nuevos espacios de trabajo, aún estaban en proceso de autorización institucional. Por otra parte, en un ajuste del plan de estudio, considerando los resultados de un proceso de autoevaluación tendiente a la acreditación de la carrera $\mathrm{ICCl}$, aparecieron nuevos requerimientos, tales como, la necesidad de mejorar las capacidades de gestión de proyectos y emprendimientos tecnológicos. El primer aspecto fue recogido a través una línea de 5 cursos de proyectos y su aplicación en otros 5 cursos de tipo desarrollo de proyectos informáticos. El aspecto de "Gestión de emprendimientos tecnológicos" se incorporó mediante la modalidad de un curso taller y vinculado los cursos "Proyectos IV" de la línea de proyectos y la "Actividad de Titulación". 
Diseño definitivo. Durante el año 2014, se concluyó el diseño de la propuesta que implementaba integralmente todos los aspectos de las bases iniciales. Sin embargo, durante el año 2015, la UTA se adjudicó un Proyecto del MINEDUC dentro del Plan de Mejoramiento Institucional para las Universidades y los Convenios de Desempeño (CD) en el marco del Fondo de Desarrollo Institucional. Específicamente, se adjudicó un CD en el área de Innovación Académica, que tenía como objetivo apoyar la gestión del cambio corporativo o institucional y ejercer un impacto significativo en el sistema de educación superior. Para ello, el CD financió aspectos como la introducción en los currículos de técnicas de aprendizaje innovadoras y centradas en los estudiantes; el fortalecimiento de la nivelación de competencias básicas de los estudiantes desfavorecidos académicamente, así como de programas que ayuden a reforzar las competencias básicas de los estudiantes en áreas como matemáticas, comprensión lectora y habilidades de estudio; la revisión y actualización de los contenidos y la duración de las carreras; el incremento de la movilidad estudiantil mediante el Sistema de Créditos Transferibles (SCT); el mejoramiento del análisis institucional; y el mejoramiento de la gestión institucional.

En este contexto, la UTA estableció nuevos estándares para los nuevos currículos para todas sus carreras. Por ejemplo, definió aspectos técnicos educativos (modelo educativo, modelo pedagógico, mecanismo de créditos, sello institucional) y aspectos de forma (modelo de proceso de diseño nuevos currículos, layout del plan de estudio). Así, desde mediados del año 2017 a mediados del año 2019, se ajustó la propuesta curricular 2014, de acuerdo a los requerimientos institucionales, según el modelo de proceso de diseño institucional y se presentó según el formato o layout definido. Cabe destacar que el perfil (competencias) y los principios rectores se mantuvieron, excepto enseñanza just-in-Time. Se incorporaron las competencias del sello institucional y se aplicó el mecanismo de cálculo de créditos transferibles.

\section{Universidad de Concepción - UdeC}

En el año 2011, se inicia oficialmente la implementación del plan de estudios orientado al desarrollo de competencias de la carrera de Ingeniería Civil Informática, luego de varios procesos internos de revisión Institucional. El plan de estudios se reduce de 12 a 11 semestres, involucra la incorporación de Sistema de Créditos Transferibles (SCT)-Chile y asignaturas con resultados de aprendizaje. En este momento, dentro del contexto de la iniciativa Nueva Ingeniería 2030, está en proceso de aprobación un nuevo plan de estudios que mantiene las características del previo, actualiza el perfil de egreso en función de las demandas del medio, incorpora las competencias sello de la institución y la arquitectura curricular definida para todas las carreras de la Facultad de Ingeniería de la Universidad, que considera un conjunto de asignaturas núcleo, más una asignatura integradora por año. Además, se definieron perfiles intermedios y se están consolidando los cuerpos académicos que cuenten con las competencias necesarias para hacerse cargo de estos aprendizajes integrados en cada año del plan de estudio, y así ir verificando el logro progresivo de los perfiles de egreso en cada uno de los estudiantes de la facultad.

El modelo educativo de la facultad está fuertemente influenciado por lo aprendido en el diseño curricular que se llevó a cabo entre el año 2002 y 2008 en la Carrera de Ingeniería Civil Informática y el Departamento de Ingeniería Informática y Ciencias de la Computación, que es el que cuenta con una mayor proporción de académicos certificados para desarrollar aprendizajes y competencias a través de aprendizaje activo y evaluación auténtica. De hecho, el proceso de rediseño de la facultad de ingeniería es liderado por la misma académica que lideró el proceso en la carrera de Ingeniería Civil Informática, por lo cual los aprendizajes de este proceso en red han impactado no sólo a un programa y sus estudiantes, sino que a las 13 especialidades y más de 3000 estudiantes de la facultad de ingeniería de la Universidad de Concepción. Los desafíos actuales son mantener las estructuras de apoyo docente (se creó el año 2016 la Unidad de Educación en Ingeniería) y promover una estructura de estímulos adecuados al mejoramiento de los aprendizajes de nuestros estudiantes, basada en evidencia y mejores prácticas de otras escuelas de ingeniería de clase mundial.

\section{Universidad Técnica Santa María - USM}

El año 2014 entró en vigencia el nuevo plan de estudios de Ingeniería Civil Informática, de 11 semestres de duración, definido a partir del trabajo realizado en los proyectos MECESUP de rediseño curricular, actualizado de acuerdo a las nuevas normas institucionales para las carreras, con relación a procesos de diseño curricular, mapa de títulos y grados, la adopción del SCT-Chile, y, recientemente, normas de competencias de sello institucional. Una característica importante a destacar del nuevo currículum, es la flexibilidad para los estudiantes, a través de 8 asignaturas electivas en los últimos semestres de la carrera, con 4 sobre temas de informática y 4 de otras disciplinas científicas o profesionales. Además, entre el tercero y el noveno semestre de la carrera, los estudiantes disponen de 7 micro asignaturas de formación integral vía actividades co-curriculares. Un tratamiento especial se debe hacer cuando un estudiante del plan de estudios antiguo cursa una asignatura equivalente del nuevo plan, con una cantidad de créditos diferente. En tales casos, los profesores acuerdan con los estudiantes actividades extras que compensen la dedicación comprometida en el plan de estudios original. 
Para la gestión y la actualización del nuevo currículum, se establecieron mecanismos conducidos por un comité académico, donde participan académicos de las áreas de especialidad. También para revisar el currículum y orientar cambios mayores se cuenta con el apoyo de un comité asesor externo formado por representantes del sector productivo, estudiantes egresados, organizaciones profesionales, y organismos del Estado. Por otro lado, para verificar los avances en las competencias, se definieron dos evaluaciones, que se realizan cuando los estudiantes han aprobado las asignaturas de los 4 primeros semestres de la carrera, que corresponde al grado de Bachiller, y cuando los estudiantes han completado las asignaturas de los 8 primeros semestres de la carrera, que corresponde al grado de Licenciatura en Ciencias de la Ingeniería. Para ambas evaluaciones, se definen problemas de tipo "integración y aplicación de conocimientos" de las materias tratadas, orientados a evaluar los resultados de aprendizaje que los estudiantes deben haber logrado, tanto a nivel individual como grupal. Esta última modalidad, se orienta a evaluar competencias de trabajo en equipo, resolución de conflictos, comunicación oral y escrita, y aspectos de ética profesional.

Una actualización curricular que deberá abordarse en el corto plazo, es la integración de las competencias de sello institucional al perfil de egreso y, junto con ello los ajustes al plan de estudios, particularmente con la redefinición de algunas competencias y resultados de aprendizaje de algunas asignaturas. La docencia orientada a la formación de competencias es un componente importante de la implementación del nuevo currículum, donde todavía hay un largo camino por recorrer. Aun cuando algunos profesores de la carrera han sido pioneros en la adopción e innovación en técnicas activas de enseñanza y de aprendizaje para formación de competencias, parte importante de los profesores aún aplican prácticas docentes tradicionales, a pesar de las oportunidades de capacitación que ofrece la institución.

\section{RESULTADOS Y DISCUSIÓN}

Los resultados de la ejecución de los dos proyectos MECESUP en red, han sido muy positivos, lográndose los objetivos planteados. La Tabla 2 resume un conjunto de indicadores relacionados con los objetivos del MECESUP, los proyectos, las Instituciones, y las carreras de las respectivas universidades de la red, los cuales son un resumen de los datos entregados por los autores en las diversas universidad y contrastados por los autores con respecto a los informes de resultados de los respectivos proyectos MECESUP mencionados anteriormente.

En primer lugar, es importante mencionar que los proyectos MECESUP, fueron concluidos lográndose los siguientes objetivos: a) Se diseñaron e implementaron nuevos currículos en las 3 universidades de la red. Cada una de ellas abordó problemas generales y específicos particulares; b) Se desarrollaron un conjunto de acciones, con apoyo de instituciones nacionales e internacionales (Canadá, Chile, México, España, USA). Estas acciones se desarrollaron a través de talleres y seminarios en conjunto con las universidades de la red y talleres en cada una de ellas. Se invitó, además, a la mayoría de las Facultades de Ingeniería del país, donde también se estuviese abordando procesos de renovación curricular, lo que permitió contrastar y socializar resultados y obtener sugerencias; c) Se puso en marcha un proceso de partida de los nuevos planes de estudio, mediante currículos de transición, que en la actualidad permite tener en ejecución un nuevo plan curricular, basado en los resultados de ambos proyectos MECEUP; y d) Cabe señalar que los logros antes mencionados corresponden al $80 \%$ de las metas propuestas en los proyectos MECESUP adjudicados, sin embargo, se observa que el logro es del 100\%, pero hay acciones que aún están en marcha y dicen relación con seguimiento y aseguramiento de calidad y revisión y actualización de los nuevos currículos, que son procesos continuos.

En segundo lugar, es importante destacar que el trabajo en red permitió romper barreras naturales que se presentan al enfrentarse a procesos de innovación, especialmente respecto a la formación por competencias, como son el temor al cambio, desconfianza con respecto al modelo y falta de entendimiento de los beneficios. Esto se vio subsanado por la movilidad estudiantil y académica dentro de la red, donde los estudiantes y profesores pudieron conocer in-situ las distintas realidades formativas y capacidades de los cuerpos académicos; también permitió compartir experiencias tanto del desarrollo del proyecto como sugerencias o buenas prácticas para su implementación y mantener una comunicación permanente de apoyo en la red.

En tercer lugar, los proyectos permitieron descubrir carencias institucionales respecto a normas y cultura organizacional para enfrentar una renovación curricular innovadora, ya sea a nivel curricular, administrativo y reglamentario. Un ejemplo es el caso del avance curricular por semestres y no por asignaturas, tema pendiente que se habían propuesto en los diseños preliminares. Otro ejemplo son las normativas específicas para regular la gestión curricular de un diseño innovador. Esto, desembocó en que se crearan unidades de apoyo al proceso formativo, institucionalizando este proceso, a través de nuevas normativas, talleres y semanarios a profesores. En cuarto lugar, es importante destacar que el país se encuentra dentro de un plan de aseguramiento de la calidad de los programas o carreras profesionales. Procesos de diseño curricular, amplios y completos, generan para las carreras involucradas exigencias adicionales que no deben ser descuidadas, ya que pasan a ser exigencias de las entidades certificadoras. Así, se requirió el diseño de procesos de autoevaluación y acreditación. 
Tabla 2: Indicadores de Impacto cualitativos de la ejecución de los Proyectos MECESUP

\begin{tabular}{|c|c|c|c|}
\hline Indicador & $\begin{array}{l}\text { Base antes de } \\
\text { ejecutar los proyectos }\end{array}$ & $\begin{array}{l}\text { Después de ejecutar los } \\
\text { proyectos }\end{array}$ & Valoración \\
\hline $\begin{array}{l}\text { Trabajo conjunto con otras } \\
\text { Universidades en Rediseño } \\
\text { curricular }\end{array}$ & Sin experiencia & $\begin{array}{l}\text { Con experiencia y red de } \\
\text { trabajo } \\
\text {. }\end{array}$ & $\begin{array}{l}\text { Como se indicó anteriormente, se desarrollaron un } \\
\text { conjunto de acciones, con apoyo de instituciones } \\
\text { nacionales e internacionales, lográndose completamente } \\
\text { este objetivo estratégico del MECESUP. }\end{array}$ \\
\hline Ejecución efectiva & $\begin{array}{l}\text { No hay datos ni } \\
\text { transparencia en el } \\
\text { uso de recursos }\end{array}$ & $\begin{array}{l}\text { Hay datos y transparencia en } \\
\text { el uso de recursos asignados }\end{array}$ & $\begin{array}{l}\text { Ya se ha señalado que se lograron el } 80 \% \text { de las metas } \\
\text { propuestas en los proyectos MECESUP, lo que es también } \\
\text { un objetivo estratégico del MECESUP. }\end{array}$ \\
\hline Acreditación de la carrera & Sin acreditación & Acreditada & $\begin{array}{l}\text { Este aspecto ha sido muy bien visto por la comunidad, } \\
\text { especialmente por los alumnos recién ingresados a la } \\
\text { carrera, ya que esta acreditación genera confianza. }\end{array}$ \\
\hline $\begin{array}{l}\text { Trabajo conjunto con otras } \\
\text { unidades internas } \\
\text { relacionadas con el proceso } \\
\text { formativo }\end{array}$ & $\begin{array}{l}\text { Descoordinación } \\
\text { y trabajo } \\
\text { disciplinario }\end{array}$ & $\begin{array}{l}\text { Coordinación y trabajo } \\
\text { interdisciplinario }\end{array}$ & $\begin{array}{l}\text { Un aprendizaje importante, dice relación con la } \\
\text { incorporación y coordinación con dichas unidades } \\
\text { académicas que normalmente cuesta incorporar a una } \\
\text { innovación curricular profunda, tal como ocurrió en este } \\
\text { caso. }\end{array}$ \\
\hline $\begin{array}{l}\text { Sistemas de apoyo al proceso } \\
\text { formativo y aseguramiento de } \\
\text { la calidad }\end{array}$ & Sin sistemas & $\begin{array}{l}\text { Con sistemas } \\
\text { institucionalizados }\end{array}$ & $\begin{array}{l}\text { Los académicos consideran este aspecto como un } \\
\text { avance positivo, ya que existe una disminución en su } \\
\text { carga de trabajo administrativo, porque los sistemas } \\
\text { permiten automatizar tareas como evaluaciones, } \\
\text { publicación de material didáctico, generación de actas } \\
\text { finales, entre otros. }\end{array}$ \\
\hline $\begin{array}{l}\text { Unidades de apoyo a los } \\
\text { procesos formativos y } \\
\text { diseños curricular }\end{array}$ & No existían & $\begin{array}{l}\text { Con unidades } \\
\text { institucionalizadas }\end{array}$ & $\begin{array}{l}\text { La Institución, considerando los resultados alcanzados en } \\
\text { los MECESUP y los objetivos del CD, procedió a la } \\
\text { creación de unidades técnicas de apoyo para toda la } \\
\text { Universidad. }\end{array}$ \\
\hline $\begin{array}{l}\text { Cuantificadores de } \\
\text { resultados (años } \\
\text { permanencia en la carrera, } \\
\text { tasas de retención, } \\
\text { repitencia, y de titulación) }\end{array}$ & $\begin{array}{l}\text { Sin } \\
\text { mediciones } \\
\text { sistemáticas }\end{array}$ & $\begin{array}{l}\text { Con medición } \\
\text { sistemática }\end{array}$ & $\begin{array}{l}\text { Se han logrado mejoras en los cuantificadores, pero } \\
\text { no alcanzando los niveles propuestos. A pesar de ello, } \\
\text { la carrera se encuentra dentro de los valores de } \\
\text { indicadores nacionales, lo cual ha permitido que se } \\
\text { mantenga acreditada. } \\
\text { En la USM la nueva carrera logró máxima acreditación }\end{array}$ \\
\hline Currículo & $\begin{array}{l}\text { Currículo } \\
\text { con } \\
\text { deficiencias }\end{array}$ & $\begin{array}{l}\text { Currículo enfrenta } \\
\text { deficiencias }\end{array}$ & $\begin{array}{l}\text { Se consideraron, en el diseño curricular, aspectos tales } \\
\text { como: excesivo tiempo de permanencia de los } \\
\text { estudiantes en las carreras, baja pertinencia de los } \\
\text { currículos en relación a las necesidades de la sociedad } \\
\text { chilena y globalizada, etc. }\end{array}$ \\
\hline $\begin{array}{l}\text { Capacidades instaladas para } \\
\text { rediseño curricular }\end{array}$ & No existían & $\begin{array}{l}\text { Autonomía y } \\
\text { experiencia }\end{array}$ & $\begin{array}{l}\text { Los académicos se muestran más capacitados para } \\
\text { innovar o actualizar el currículo en el tiempo, lo que } \\
\text { permite reaccionar con mayor rapidez a los cambios } \\
\text { que requiere el medio productivo. }\end{array}$ \\
\hline $\begin{array}{l}\text { Capacidades académicas en } \\
\text { pedagogías activas }\end{array}$ & Sin capacidades & Con capacidades & $\begin{array}{l}\text { Se realizaron talleres y seminarios, en conjunto con las } \\
\text { universidades de la red y talleres en cada una de ellas. } \\
\text { En varios seminarios, se invitó a la mayoría de las } \\
\text { facultades de ingeniería del país, en las cuales también } \\
\text { se estuviese abordando procesos de renovación } \\
\text { curricular, lo que permitió contrastar y socializar } \\
\text { resultados y obtener sugerencias. }\end{array}$ \\
\hline $\begin{array}{l}\text { Duración del programa } \\
\text { de la carrera }\end{array}$ & 6 años & $\begin{array}{l}5,5 \text { años incluida la } \\
\text { articulación con Magíster }\end{array}$ & $\begin{array}{l}\text { Este aspecto aún se encuentra en proceso de } \\
\text { evaluación, ya que la primera cohorte de este proceso } \\
\text { no ha egresado. }\end{array}$ \\
\hline $\begin{array}{l}\text { Sin mecanismos para la } \\
\text { revisión y actualización del } \\
\text { currículo }\end{array}$ & No existían & $\begin{array}{l}\text { Con unidades } \\
\text { institucionalizadas }\end{array}$ & $\begin{array}{l}\text { Este aspecto aún se encuentra en proceso de } \\
\text { evaluación, ya que la primera cohorte de este proceso } \\
\text { no ha egresado y será ejecutado por unidades } \\
\text { institucionalizadas. }\end{array}$ \\
\hline Perfil de egreso & $\begin{array}{l}\text { Ad-hoc, sin considerar } \\
\text { usuarios }\end{array}$ & $\begin{array}{l}\text { Orientado a } \\
\text { competencias, levantado } \\
\text { a partir de usuarios clave. } \\
\end{array}$ & $\begin{array}{l}\text { Usuarios clave consideraron como una mejora positiva } \\
\text { su inclusión en el diseño del perfil de egreso y señalan } \\
\text { que su participación debe ser permanente. }\end{array}$ \\
\hline Proceso formativo & $\begin{array}{l}\text { Clásico, basado en } \\
\text { método de la clase } \\
\text { magistral }\end{array}$ & $\begin{array}{l}\text { Orientado por los } \\
\text { resultados de } \\
\text { aprendizaje, y } \\
\text { aprendizaje basado en } \\
\text { problemas y proyectos } \\
\text { (ABP y P). }\end{array}$ & $\begin{array}{l}\text { Los estudiantes consideran que el proceso formativo ha } \\
\text { mejorado capacidades con las que no contaban en el } \\
\text { modelo anterior. En particular, consideran que los } \\
\text { proyectos ayudan a su desarrollo profesional. }\end{array}$ \\
\hline
\end{tabular}


Tabla 2: continuación.

\begin{tabular}{|l|l|l|l|}
\hline Indicador & $\begin{array}{l}\text { Base antes de } \\
\text { ejecutar los proyectos }\end{array}$ & $\begin{array}{l}\text { Después de ejecutar los } \\
\text { proyectos }\end{array}$ & Valoración \\
\hline Competencias & $\begin{array}{l}\text { No orientado a } \\
\text { competencias }\end{array}$ & $\begin{array}{l}\text { Competencias } \\
\text { Específicas y genéricas, } \\
\text { particularmente en } \\
\text { emprendimiento y } \\
\text { liderazgo. }\end{array}$ & $\begin{array}{l}\text { Los estudiantes que cursaron asignaturas donde se } \\
\text { desarrollaron competencias tales como emprendimiento } \\
\text { y liderazgo, resaltaron su importancia para su futuro } \\
\text { profesional y su capacidad de autoempleo. }\end{array}$ \\
\hline Movilidad Estudiantil & $\begin{array}{l}\text { Sin convenios de } \\
\text { movilidad }\end{array}$ & Con convenios de movilidad & $\begin{array}{l}\text { Hubo una alta movilidad estudiantil. Por ejemplo, sobre 100 } \\
\text { alumnos (UTA), fueron favorecidos por convenios } \\
\text { específicos con otras instituciones de educación superior. } \\
\text { Ha sido considerado como un aspecto positivo y que debe } \\
\text { perdurar en el tiempo. }\end{array}$ \\
\hline Infraestructura & Ad-hoc & $\begin{array}{l}\text { Para el desarrollo de } \\
\text { competencias, en específico } \\
\text { para proyectos. }\end{array}$ & $\begin{array}{l}\text { Nuevos y mejores laboratorios y equipamiento } \\
\text { computacional, orientados al desarrollo de competencias, } \\
\text { que han sido recibidos de manera positiva por los alumnos } \\
\text { y académicos. Creación y adecuación de infraestructura, tal } \\
\text { como un laboratorio para proyectos. }\end{array}$ \\
\hline $\begin{array}{l}\text { Material didáctico para } \\
\text { desarrollo de competencias } \\
\text { y proyectos }\end{array}$ & No existían & $\begin{array}{l}\text { Material creado y registrado en } \\
\text { biblioteca institucional }\end{array}$ & $\begin{array}{l}\text { Generación de material didáctico ad-hoc que se registra en } \\
\text { biblioteca y sistemas informáticos }\end{array}$ \\
\hline $\begin{array}{l}\text { Uso de TICs para desarrollo } \\
\text { de competencias y } \\
\text { proyectos }\end{array}$ & No se usaban & $\begin{array}{l}\text { TICs (software) para } \\
\text { proyectos, formación en } \\
\text { trabajo en equipo, etc. }\end{array}$ & $\begin{array}{l}\text { Uso intensivo de TICs en los procesos educativos y de } \\
\text { gestión curricular. }\end{array}$ \\
\hline
\end{tabular}

En quinto lugar, dado que las carreras de ingeniería en la institución y de la red son impartidas por diferentes unidades disciplinares, se hizo necesario asegurar distintos procesos para que dichas unidades puedan trabajar en conjunto de manera satisfactoria. Un aprendizaje importante dice relación con la incorporación y coordinación con dichas unidades académicas, que normalmente se oponen a la innovación curricular profunda, tal como se planteó en este caso. Los aspectos citados anteriormente como los son el just in time o el avance curricular por semestres, fueron cuestionados o hasta rechazados. Cabe señalar que, a juicio de los autores, tanto la enseñanza just in time como el avance curricular podrían haberse implementado si las autoridades superiores respectivas hubieran accedido a generar la normativa necesaria para llevarla a cabo, dado que tanto las condiciones de infraestructura como académicas se encontraban presentes en las unidades respectivas. Además, otro aspecto necesario para dichos cambios, sería la autonomía de la carrera en cuanto a los procesos de enseñanza, es decir, no depender o depender al mínimo de departamentos externos a la Facultad de Ingeniería correspondiente. Detalles del perfil de egreso propuesto se encuentran en la presentación "Diseño e implantación de un currículo dirigido por competencias, integrado-estructurado alrededor de temas, orientado a proyectos y con una enseñanza de tipo Just-In-Time (JITT)" disponible en http://sochedi2013.pucv.cl/05_programa.htm.

En sexto lugar, cabe destacar el aprendizaje, tanto de las unidades ejecutoras como institucionales, para diseñar nuevos currículos en el futuro. Hoy es posible mantener una autonomía para tales efectos que significan ahorro de recursos tales como tiempo, diseño y humano para mantener actualizados o renovados los currículos en ingeniería de la red. Considerando los puntos anteriormente analizados, se puede apreciar que las medidas ejecutadas, desde el punto de vista cualitativo, tuvieron un impacto positivo en la formación de los alumnos de Ingeniería Informática de las universidades de la red.

\section{CONCLUSIONES}

De acuerdo al trabajo presentado y a los resultados obtenidos, se pueden plantear las siguientes conclusiones principales:

1) A nivel curricular, se puede señalar que la renovación profunda e innovadora, requiere de acciones claves, como la definición de un perfil con base en competencias, validado interna y externamente, y un diseño curricular, creado con el apoyo asesorías internacionales. La formación y capacitación de los académicos, tanto en el diseño y la filosofía subyacente del currículo, fue imprescindible. Sin embargo, un perfil validado y un diseño de calidad, son condiciones necesarias, pero no suficientes. Se debe planificar y ejecutar la implantación, que consideró aspectos como el desarrollo e implementación de sistemas informáticos de soporte, la configuración de espacios de trabajo, el logro del compromiso y apoyo institucional, creación de unidades técnicas y mecanismos para la revisión y actualización del currículo. Finalmente, un aspecto clave es la ejecución de un plan de estudios piloto, para corroborar las hipótesis planteadas y anticipar dificultades prácticas imprevistas, ya que fallas en esos dos aspectos, pueden truncar todo el esfuerzo realizado. Se lograron importantes aprendizajes para enfrentar el diseño definitivo del próximo plan de estudios, a realizarse en años venideros. 
2) A nivel de la red e instituciones (UTA, UdC, USM), es importante destacar que los proyectos MECESUP permitieron enfrentar los desafíos de innovación curricular; facilitar movilidad estudiantil y académica; permitieron compartir experiencias, tanto del desarrollo del proyecto como sugerencias o buenas prácticas para su implementación; y mantener una comunicación permanente de colaboración entre las instituciones de la red.

3) Con respecto a los objetivos del MINEDUC y MECESUP, éstos se cumplieron, por cuanto las carreras pasaron desde un proceso formativo clásico a uno orientado por competencias, donde lograron la capacidad para ejecutar el rediseño, orientación a la calidad (acreditación), capacidad de cambio institucional, que desencadenó en la creación de unidades técnicas de apoyo y nuevas normativas.

4) Finalmente, es importante mencionar que estos cambios y mejoras pueden llevarse a cabo en otros países, principalmente en la Macro Región Centro sur Andina, que presenta una realidad laboral y académica similar al Norte de Chile. Los resultados obtenidos pueden servir para intercambiar experiencias con otras instituciones, dedicadas a la formación de ingenieros y específicamente de ingenieros informáticos.

\section{AGRADECIMIENTOS}

Este trabajo de cuenta con el financiamiento del programa de Investigación de la Universidad de Tarapacá, Arica, Chile (2017-2018), en el contexto de los proyectos, código 8742-17 y código 8727-18. Además, un especial agradecimiento a los directores de los proyectos por todo su apoyo en la recolección de la información, evidencias y hechos documentados en este artículo.

\section{REFERENCIAS}

Bédard, D., Lison, C., y otros 3 autores; Problem-based and project-based learning in engineering and medicine: determinants of students' engagement and persistence. Interdisciplinary Journal of Problem-based Learning, 6(2), 7-30 (2012).

Bédard, D., Louis, R., Bélisle, M. y Viau, R.; Problem-and Project-Based Learning in Engineering at the University of Sherbrooke: Impact on Students' and Teachers' Perceptions, Management of Change, 109-128. (2007).

Crawley, E., Malmqvist, J., Lucas, W. y Brodeur, D.; The CDIO syllabus v2. 0. An updated statement of goals for engineering education, Proceedings of 7th international CDIO conference, Copenhagen, Denmark, 1-42 (2011).

Ellahi, R., Khan, M. y Shah, A.; Redesigning Curriculum in line with Industry 4.0, Procedia computer science, 151, 699-708 (2019).

García-Blanco, M., y Sempértegui, E., La inserción laboral en la Educación Superior. La perspectiva latinoamericana, Educación XX1, 21(2), 323-347 (2018).

George, A; Case studies and theory development: The method of structured, focused comparison. In Alexander L. George: A pioneer in political and social sciences. Springer, Cham, 191-214 (2019).

Herrero, E., Cervero, A., Esteban, M., y Bernardo, A., ¿Por qué abandonan los alumnos universitarios? Variables de influencia en el planteamiento y consolidación del abandono, Educación XX1, 21(2), 131-154 (2018).

Hurtubise, L., y Roman, B.; Competency-based curricular design to encourage significant learning, Current Problems in Pediatric and Adolescent Health Care, 44(6), 164-169 (2014).

Icarte, G. y Labate, H., Metodología para la Revisión y Actualización de un Diseño Curricular de una Carrera Universitaria Incorporando Conceptos de Aprendizaje Basado en Competencias, Formación universitaria, 9(2), 03-16 (2016).

Jensen, I., y Lassen, I.; Redesigning the curriculum: applying problem based learning in a new context. Pedagogy, Culture \& Society, 28(1), 37-57 (2020).

Kim, K., Kim, J., Yoon, G. y Woo, H.; University Curriculum Development and Management Based on Korean National Competency Standards: A Case Study. Journal of Technical Education and Training, 11(1), 1-10 (2019).

Luna-García, H., Álvarez-Rodríguez, F. y Mendoza-González, R., Modelo de gestión para diseño curricular basado en prácticas de ingeniería de software, Revista Investigación Educativa, 17(3), 61-78 (2015).

Marsh, S., Paine, M. y Gibson, K.; Redesigning a Comprehensive Integrated Pharmacology Course for Active Learning in a Competency-Based Curriculum. The FASEB Journal, 31(1_supplement), 576-12 (2017).

Martin, A., Martin, D., Aguilera, I. y otros 5 autores, Nuevas combinaciones de aula inversa con just in time teaching y análisis de respuestas de alumnos, RIED. Revista Iberoamericana de Educación a Distancia, 21(1), 175-194 (2018).

Martínez, E., Fonseca, R. y Tapia, H. Implementación de Rediseños Curriculares Universitarios en Educación, una Tarea Compleja, Formación Universitaria, 12(3), 55-66 (2019).

Novak, D., Hallowell, R., y otros 2 autores; Continuum of innovation: Curricular renewal strategies in undergraduate medical education, 2010-2018, Academic Medicine, 94(11S), 79-85 (2019). 
Pinto, M., Jung, H. y Sudbrack, E., Políticas educacionales brasileñas: consecuencias de un currículo nacional. Revista de Investigación Educativa, 21(2), 1-9 (2019).

Rangel, H., Una mirada internacional de la construcción curricular. Por un currículo vivo, democrático y deliberativo, Revista de Investigación Educativa, 17(1), 1-16 (2015).

Rata, E.; Knowledge-rich teaching: A model of curriculum design coherence, British Educational Research Journal, 45(4), 681-697 (2019).

Romero, I. y González, A., Integración curricular del perfil por competencias a partir de un ordenamiento transversal, Opción, 32(13), 411-434 (2016).

Ryan, T., Grimes, T., y otros 5 autores; Design and implementation of an integrated competency-focused pharmacy programme: a case report, Pharmacy, 7(3), 121 (2019).

Tejada-Fernández, J. y Ruiz-Bueno, C., Evaluación de competencias profesionales en educación superior: retos e implicaciones, Educación XXI, 19(1), 17-38 (2016).

Tarrats-Pons, E., Arimany-Serrat, N., Arando-Lasagabaster, S., Martínez-González, M., Samonneau, M., Pilniere, V., y Mathieu, L., Educación superior en alternancia transfronteriza entre Francia y España, Educación XX1, 21(2), 275-300 (2018).

Tracy, S., Qualitative research methods: Collecting evidence, crafting analysis, communicating impact, John Wiley \& Sons (2019).

Trujillo, A., y de Justo Moscardó, E., Evaluación del diseño, proceso y resultados de una asignatura técnica con aprendizaje basado en problemas, Educación XX1, 21(2), 179-203 (2018).

Vincent, S. y Focht, W.; Interdisciplinary environmental education: elements of field identity and curriculum design, Journal of Environmental Studies and Sciences, 1, 14-35 (2011). 
